\title{
Article \\ Genome-Wide Identification and Expression Profiling of the BZR Transcription Factor Gene Family in Nicotiana benthamiana
}

\author{
Xuwei Chen ${ }^{1}$, Xinyang Wu ${ }^{1,2}$, Shiyou Qiu ${ }^{1}$, Hongying Zheng ${ }^{1}$, Yuwen Lu ${ }^{1}$, Jiejun Peng ${ }^{1}$, Guanwei Wu ${ }^{1}$, \\ Jianping Chen ${ }^{1}\left(\mathbb{D}\right.$, Shaofei Rao ${ }^{1, *}$ and Fei Yan ${ }^{1, *}$
}

1 State Key Laboratory for Managing Biotic and Chemical Threats to the Quality and Safety of Agro-Products, Key Laboratory of Biotechnology in Plant Protection of Ministry of Agriculture and Zhejiang Province, Institute of Plant Virology, Ningbo University, Ningbo 315211, China; 1811075003@nbu.edu.cn (X.C.); xy_wu@zju.edu.cn (X.W.); qiushiyoudyzx@126.com (S.Q.); zhenghongying@nbu.edu.cn (H.Z.); luyuwen@nbu.edu.cn (Y.L.); pengjiejun@nbu.edu.cn (J.P.); wuguanwei@nbu.edu.cn (G.W.); jianpingchen@nbu.edu.cn (J.C.)

2 College of Life Science, China Jiliang University, Hangzhou 310058, China

* Correspondence: raoshaofei@nbu.edu.cn (S.R.); yanfei@nbu.edu.cn (F.Y.)

check for updates

Citation: Chen, X.; Wu, X.; Qiu, S.; Zheng, H.; Lu, Y.; Peng, J.; Wu, G.; Chen, J.; Rao, S.; Yan, F. GenomeWide Identification and Expression Profiling of the BZR Transcription Factor Gene Family in Nicotiana benthamiana. Int. J. Mol. Sci. 2021, 22, 10379. https://doi.org/10.3390/ ijms221910379

Academic Editor: Bartolome Sabater

Received: 23 August 2021

Accepted: 23 September 2021

Published: 26 September 2021

Publisher's Note: MDPI stays neutral with regard to jurisdictional claims in published maps and institutional affiliations.

Copyright: (c) 2021 by the authors. Licensee MDPI, Basel, Switzerland. This article is an open access article distributed under the terms and conditions of the Creative Commons Attribution (CC BY) license (https:/ / creativecommons.org/licenses/by/ $4.0 /)$.
Abstract: Brassinazole-resistant (BZR) family genes encode plant-specific transcription factors (TFs), play essential roles in the regulation of plant growth and development, and have multiple stressresistance functions. Nicotiana benthamiana is a model plant widely used in basic research. However, members of the BZR family in N. benthamiana have not been identified, and little is known about their function in abiotic stress. In this study, a total of 14 BZR members were identified in the N. benthamiana genome, which could be divided into four groups according to a phylogenetic tree. NbBZRs have similar exon-intron structures and conserved motifs, and may be regulated by cis-acting elements such as STRE, TCA, and ARE, etc. Organ-specific expression analysis showed that NbBZR members have different and diverse expression patterns in different tissues, and most of the members are expressed in roots, stems, and leaves. The analysis of the expression patterns in response to different abiotic stresses showed that all the tested $N b B Z R$ members showed a significant down-regulation after drought treatment. Many $N b B Z R$ genes also responded in various ways to cold, heat and salt stress treatments. The results imply that NbBZRs have multiple functions related to stress resistance.

Keywords: Brassinazole-resistant (BZR) transcription factor; Nicotiana benthamiana; genome-wide expression analysis; abiotic stress

\section{Introduction}

The plant steroid hormones, Brassinosteroids (BRs), play an important role in regulating plant growth and development, and biotic and abiotic stress responses [1]. In the past 20 years, the signal transduction pathway of BR has been well resolved [2-5]. The BR receptor Brassinosteroid insensitive 1 (BRI1) is a leucine-rich, repeated, receptor-like kinase (LRR RLK) located in the plasma membrane. When BR molecules are detected, it recruits another LRR RLK BRI1- associated receptor kinase (BAK1) to form a receptor complex. The fully activated BRI1/BAK1 complex then triggers a series of downstream phosphorylation events, which ultimately lead to the regulation of a large number of genes [2,6-8]. Brassinazole resistant 1 (BZR1) and Brassinosteroid insensitive 1-ethyl methanesulfonate-suppressor 1 (BES1) are two downstream transcription factors (TFs) belonging to the Brassinazoleresistant (BZR) TF family. They can be dephosphorylated by protein phosphatase when BR is detected [9]. Dephosphorylated BZR1 and BES1 accumulate in the nucleus and directly bind to cis-elements to regulate plant growth and development [10].

The BZR gene family encodes a novel, plant-specific TF, which can directly mediate and regulate BR signaling [11]. In Arabidopsis, the BZR gene family includes BZR1, BES1, 
and BES1/BZR1 homolog1 (BEH1)-BEH4, which are highly similar to BZR1/BES1 [8,10,12]. Both BZR1 and BES1 have a basic helix-loop-helix (bHLH) DNA binding motif at the $\mathrm{N}$-terminus, which is a highly conserved domain in the entire family, but their functions are different [12]. BZR1 binds to a BR response element (CGTGT/CG motif) to inhibit the BRbiosynthetic gene expression [13], while BES1 binds to the E box (CANNTG sequence) to activate BR-induced gene expression [14]. In addition to the highly conserved N-terminal domain, the central region of the BZR protein usually has 22-24 predicted Glycogen synthase kinase-3 (GSK3) family phosphorylation sites. Some proteins also contain putative PEST motifs involved in protein degradation [12].

The tolerance of plants to adversity, such as water stress, temperature, or changes in soil salinity, depends on their ability to switch between growth activation and inhibition under adverse conditions $[15,16]$. The abscisic acid (ABA) signaling pathway is a key method to control environmental stress responses $[17,18]$ but it appears that BRs mediate the adaptation to drought, cold, heat, and salinity either by crosstalk with the ABA pathway or independently of it. The regulation of the BZR transcription factors in Arabidopsis is an important point of convergence between $B R$ and stress responses $[1,19]$.

Several mechanisms that inhibit BES1 activity during stress are described. Under drought stress, BES1 inhibits BR-regulated growth through DSK2-mediated autophagy degradation [20]. In addition to regulating the abundance of the BES1 protein, drought also affects BES1 activity through its interaction with Responsive to desiccation 26 (RD26). RD26 is transcriptionally induced during drought, and then RD26 inhibits BES1 and promotes the drought response. At the mechanistic level, these two TFs may interact to form a heterodimer, which synergistically binds to G-Box (CACGTG) promoter elements. BES1 and RD26 have opposing activities, and RD26 inhibits BES1 activity by interacting with BES1 on a common promoter element [21,22]. TINY is an Apetala2/ethylene responsive factor TF. In drought stress, TINY is also induced to antagonize BES1, inhibit the growth regulated by BR and promote the expression of drought-responsive genes [23]. WRKY46, WRKY54 and WRKY70 can directly interact with BES1 to promote BR-regulated plant growth, while suppressing drought-induced, genome-wide transcripts to inhibit drought tolerance. However, the protein level of WRKY54 decreases during drought stress to induce drought tolerance in plants. Therefore, the degradation of BES1-interacting transcription factors during drought is another mechanism through which plants respond to drought [24].

Recent studies also show that BZR transcription factors are involved in regulating growth response when the temperature fluctuates. Low temperature treatment can also induce the dephosphorylation of BZR1 and BES1. The unphosphorylated forms of BZR1 and BES1 can promote the expression of C-repeat/dehydration-responsive element binding factor1 (CBF1) and CBF2, which positively regulates cold-stress responses. The study by $\mathrm{Li}$ et al. showed that, under non-domesticated and domesticated conditions, the overexpression of BIN2 could cause a hypersensitivity to freezing stress, while the triple mutants of bin2-3 bil1 bil2, and the gain-of-function mutants bzr1-1D and bes1-D, have an enhanced tolerance to freezing stress [25]. When the temperature increases, BZR1 accumulates in the nucleus and induces the expression of growth promoting genes, which can regulate thermalmorphogenesis directly or by binding to the promoter of Phytochrome interacting factor 4 (PIF4) [26,27].

High salt can also cause root growth arrest by inhibiting the accumulation of BZR1 in the nucleus and the subsequent BR signaling function [28]. The exogenous application of BR can help plants cope better with high salt conditions by regulating the output of BR and ethylene signals [19].

BZR TF is also shown to play an important role in participating in multiple stress response pathways in other species. In wheat, the BES/BZR family transcription factor TaBZR2 plays an active role in drought response by activating T. aestivum Glutathione S-transferase-1 (TaGST1) and mediates the dialogue between BR and drought signaling pathways [29]. Li et al. found that the BZR gene in legumes can significantly respond to 
drought and salt stress [30]. In Brassica rapa, members of the BrBZR TF family may be involved in the regulation of stress-related activities [31]. Manoli et al. found that maize BZR TFs not only play a role in regulating plant physiology and morphology, but also have stress signal activity [32]. Thus, these transcription factors play an active role in the BR signal transduction in many plants. However, members of the BZR TF family in the important experimental plant, Nicotiana benthamiana, have not been identified, and their related functions in stress resistance are unreported. In this study, we systematically identified BZR members in N. benthamiana, analyzed their expression patterns in different tissues and their gene expression profiles in response to drought, cold, heat, and salt stress. Our work lays the foundation for the future functional analysis of the BZR gene in $N$. benthamiana, and provides valuable information for a further understanding of the structure and expression of the BZR gene in solanaceous crops.

\section{Results}

\subsection{Identification of BZR Gene Family Members in N. benthamiana}

The BZR transcription factor contains a BES1_N superfamily domain, and we identified possible BZR members in N. benthamiana based on this standard. The amino acid sequences of the six identified Arabidopsis BZR members were downloaded from The Arabidopsis Information Resource (https:/ / www.arabidopsis.org/ accessed on 22 September 2021), and the N. benthamiana genome sequence was downloaded from Sol Genomics Network (https://solgenomics.net/ accessed on 22 September 2021). After two rounds of BLASTP, a total of 14 NbBZRs were identified (Supplementary Table S1). These members were named from NbBZR1 to NbBZR14 according to their chromosomal positions. They ranged in length from 182 to 330 amino acids (aa) and most were about 300 aa long. Their calculated molecular weights were $20-35 \mathrm{kDa}$, and their isoelectric points were in the range from 6.89-9.39 (Table 1). 
Table 1. Detailed information of the 14 predicted BZR proteins in N. benthamiana.

\begin{tabular}{|c|c|c|c|c|c|c|c|c|}
\hline Gene Symbol & Gene Locus & Gene Position & Chromosome Location & Strand & CDS (bp) & $\begin{array}{l}\text { Protein Length } \\
\text { (aa) }\end{array}$ & $\begin{array}{c}\text { Molecular } \\
\text { Weight(kDa) }\end{array}$ & Theoretical PI \\
\hline NbBZR1 & Niben101Scf00073g05010.1 & Niben101Scf00073 & Niben101Scf00073:539113,545181 & + & 981 & 327 & 35.27 & 7.65 \\
\hline NbBZR2 & Niben101Scf00219g06001.1 & Niben101Scf00219 & Niben101Scf00219:603740,606595 & + & 990 & 330 & 35.27 & 8.44 \\
\hline NbBZR3 & Niben101Scf00894g00004.1 & Niben101Scf00894 & Niben101Scf00894:692,3575 & - & 966 & 322 & 34.59 & 9.05 \\
\hline NbBZR4 & Niben101Scf01983g15010.1 & Niben101Scf01983 & Niben101Scf01983:1485193,1491375 & - & 987 & 329 & 35.43 & 9.39 \\
\hline NbBZR5 & Niben101Scf03110g05009.1 & Niben101Scf03110 & Niben101Scf03110:623795,626566 & + & 984 & 328 & 35.08 & 9.34 \\
\hline NbBZR6 & Niben101Scf03282g02006.1 & Niben101Scf03282 & Niben101Scf03282:277240,278755 & + & 720 & 240 & 24.74 & 9.37 \\
\hline NbBZR7 & Niben101Scf03729g00010.1 & Niben101Scf03729 & Niben101Scf03729:20537,23061 & - & 597 & 199 & 20.59 & 9.33 \\
\hline NbBZR8 & Niben101Scf04132g01002.1 & Niben101Scf04132 & Niben101Scf04132:171903,178765 & + & 984 & 328 & 35.70 & 7.58 \\
\hline NbBZR10 & Niben101Scf05948g02026.1 & Niben101Scf05948 & Niben101Scf05948:259103,263853 & - & 975 & 325 & 35.10 & 8.44 \\
\hline NbBZR11 & Niben101Scf06112g01006.1 & Niben101Scf06112 & Niben101Scf06112:109855,111842 & + & 969 & 323 & 34.85 & 9.16 \\
\hline NbBZR12 & Niben101Scf10412g00006.1 & Niben101Scf10412 & Niben101Scf10412:11951,15403 & - & 693 & 231 & 24.34 & 6.89 \\
\hline NbBZR13 & Niben101Scf10887g01009.1 & Niben101Scf10887 & Niben101Scf10887:126261,127150 & + & 546 & 182 & 20.79 & 8.47 \\
\hline NbBZR14 & Niben101Scf12841g03023.1 & Niben101Scf12841 & Niben101Scf12841:315425,319633 & + & 948 & 316 & 33.93 & 9.18 \\
\hline
\end{tabular}

Notes: The signs " + " and " -" signify sense and anti-sense transcription respectively. 


\subsection{Phylogenetic Analysis of NbBZR Genes}

In order to understand the relationships between the BZR family genes in Arabidopsis and $N$. benthamiana, a phylogenetic tree was constructed using the amino acid sequences of the six Arabidopsis and fourteen N. benthamiana members. The fourteen NbBZR genes could be divided into four groups, with five members in group I (NbBZR2, -12, -5, -3, -11), which also contained AtBES1, AtBZR1, AtBEH1, and AtBEH2 (Figure 1). NbBZR6 and $N b B Z R 7$ form the second group (Figure 1). There are five members in group III (NbBZR4, $-14,-8,-1,-10$ ), and AtBEH3, AtBEH4 are located in this group (Figure 1). Two members were contained in group IV, including NbBZR9 and NbBZR13, which do not contain an Arabidopsis homolog (Figure 1).

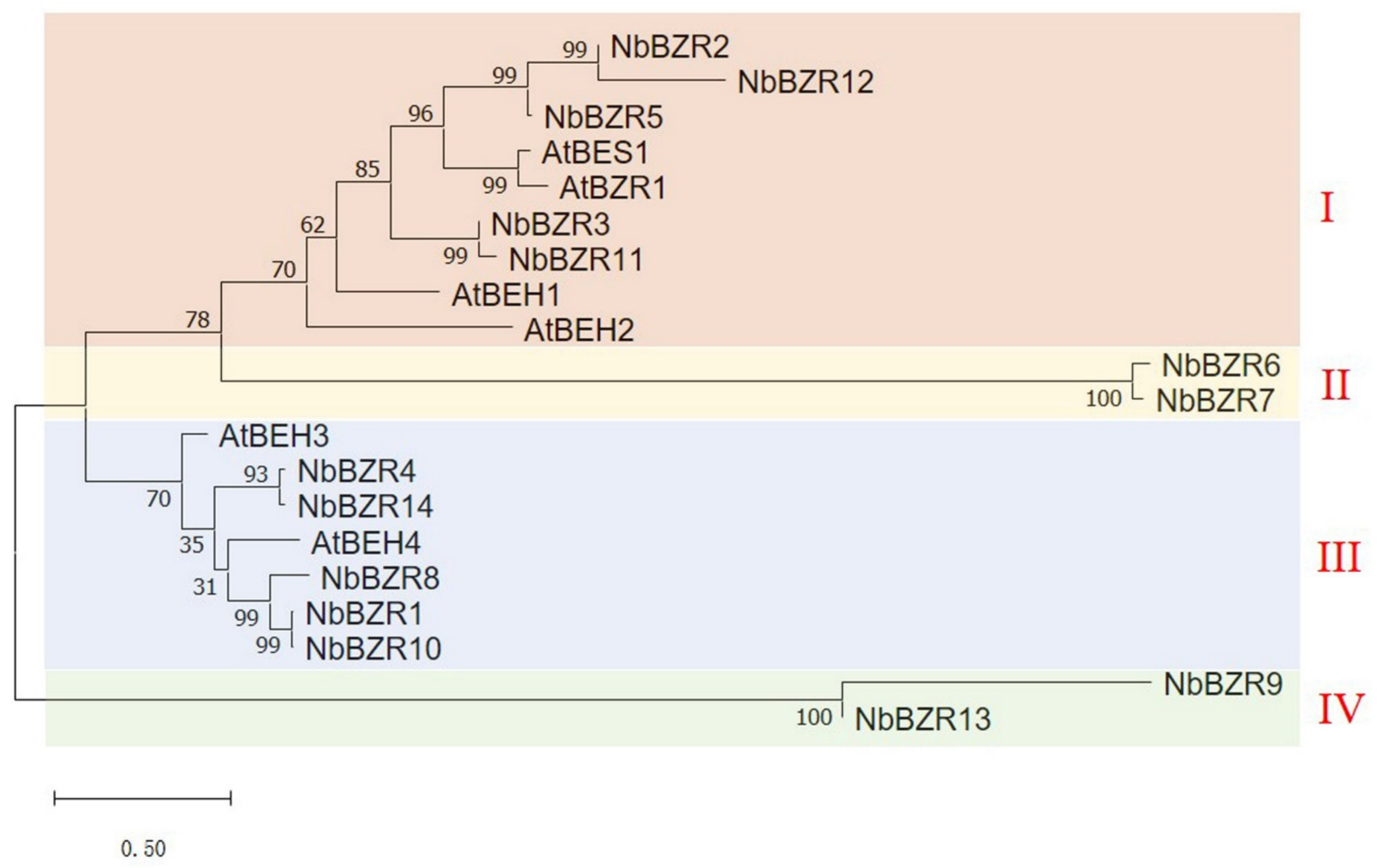

Figure 1. Phylogenetic tree of BZR transcription factors in N. benthamiana and A. thaliana. The phylogenetic tree was constructed using BZR amino acid sequences by the neighbor-joining method in MEGA X with 1000 bootstrap replicates. The phylogenetic tree was divided into four groups, which were shown in different colors, and identified by red Roman numerals.

\subsection{Analysis of NbBZR Conserved Motifs, Gene Structure and Functional Domains}

An online MEME analysis was next used to identify conserved motifs among the $14 \mathrm{NbBZR}$ members, and a total of 10 conserved motifs were predicted (Supplementary Table S2). Most members contained 5-9 motifs, but NbBZR6/-7/-9/-13 contained only two (Figure 2A,B). All 14 members contained the conserved BES1_N functional domain and most of them had two exons (Figure 2C).

\subsection{Prediction of Cis-Elements in the NbBZR Promoters}

In order to further understand the possible regulatory mechanism of the NbBZR gene, we used the PlantCARE web server to search for possible cis-elements in the NbBZR 2000 bp promoter region. A total of 2164 cis-acting elements of 84 types were identified in the NbBZR promoter region (Supplementary Table S3). These cis-acting elements were related to environmental stress, hormonal response, development and light response, etc. (Figure 3A). A total of 101 elements related to environmental stress in the whole family were predicted in 10 categories, of which STRE, TCA and ARE elements were the most abundant (Figure 3B). A total of 187 hormone-related components were predicted in 13 categories, 
mainly related to JA, ethylene, and ABA (Figure 3C). This indicated that the NbBZR family genes may have been involved in a variety of stress and plant hormone response processes, and could effectively promote plant growth and stress resistance.

A

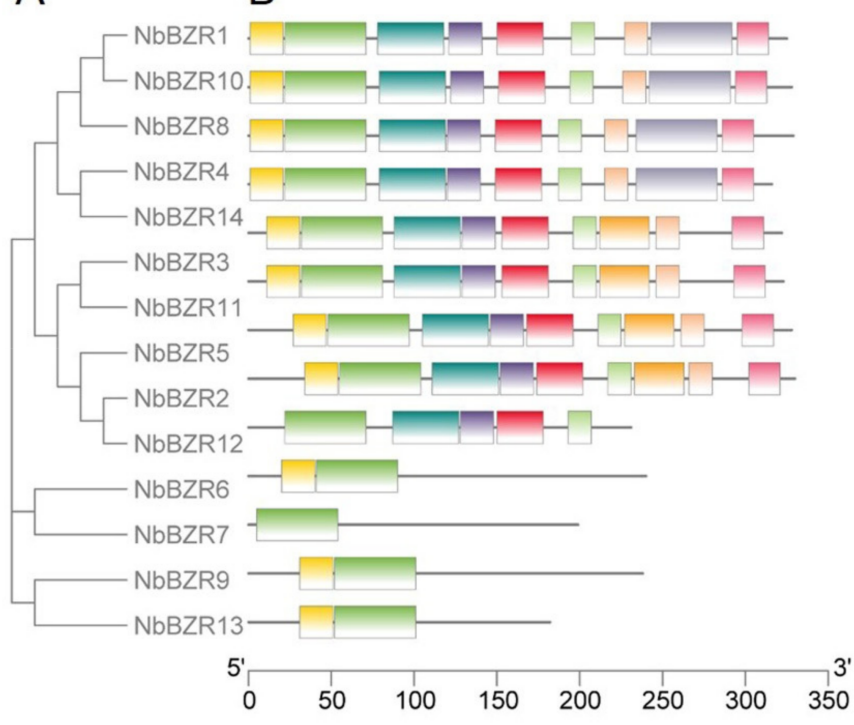

C

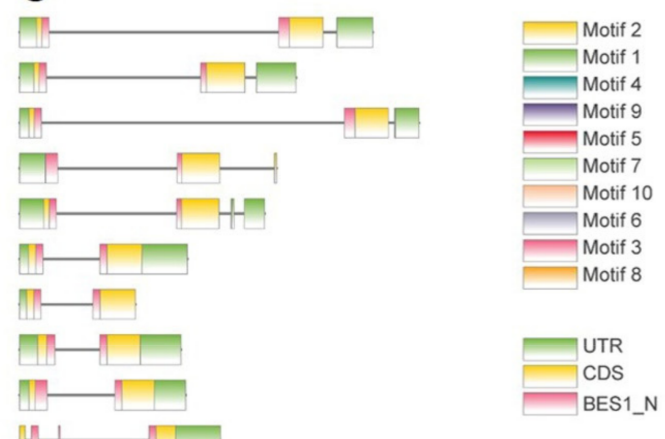

Figure 2. Analysis of conserved motifs, gene structure and domains in the BZR genes of N. benthamiana. (A) Phylogenetic tree constructed using the NbBZR protein sequences. (B) Ten types of conserved motifs are predicted in the NbBZR protein sequences. The different motifs are shown in different color boxes. The sequence information for each motif is provided in Supplementary Table S2. (C) The gene structure of NbBZR members (untranslated regions, exons, and introns are shown as light green boxes, yellow boxes and horizontal lines, respectively. The red boxes represent the BES1_N domain).
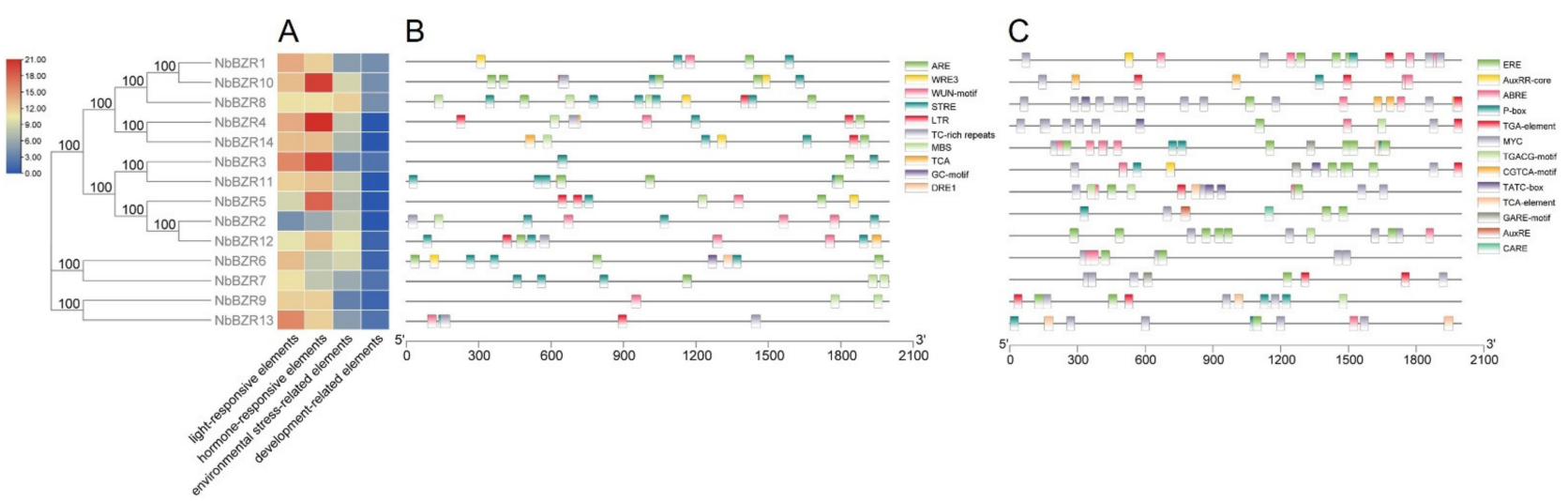

Figure 3. Prediction of cis-acting elements in the NbBZR promoter regions. (A) Schematic representation of the numbers of four types of cis-acting elements predicted in the promoter region of each NbBZR member. (B,C) The type, quantity and position of environmental stress-related elements $(\mathbf{B})$ and hormone-response elements $(\mathbf{C})$ in the $N b B Z R$ promoter region.

\subsection{Expression Patterns of NbBZR Genes in Different Tissues}

We then analyzed the expression level of most $N b B Z R s$ in five different tissues (roots, stems, young leaves, mature leaves and flowers). The results from NbBZR9/-12/-13 were not presented because their expression levels proved to be very low. NbBZR5 was the only member that was highly expressed in mature leaves (Figure $4 \mathrm{~A}, \mathrm{~K}$ and Supplementary Figure S1). $N b B Z R 4 /-7 /-8 /-14$ were expressed at high levels in the stem, especially NbBZR7 (Figure 4A,K and Supplementary Figure S1). NbBZR1/-3/-4/-5/-7/-8/10/-11/-14 were expressed at high levels in the roots but, except for $N b B Z R 10$, the $N b B Z R$ transcripts did not accumulate to high levels in flowers (Figure 4A,K and Supplementary Figure S1). These results indicated that the different $\mathrm{NbBZRs}$ were expressed differently in different tissues. 


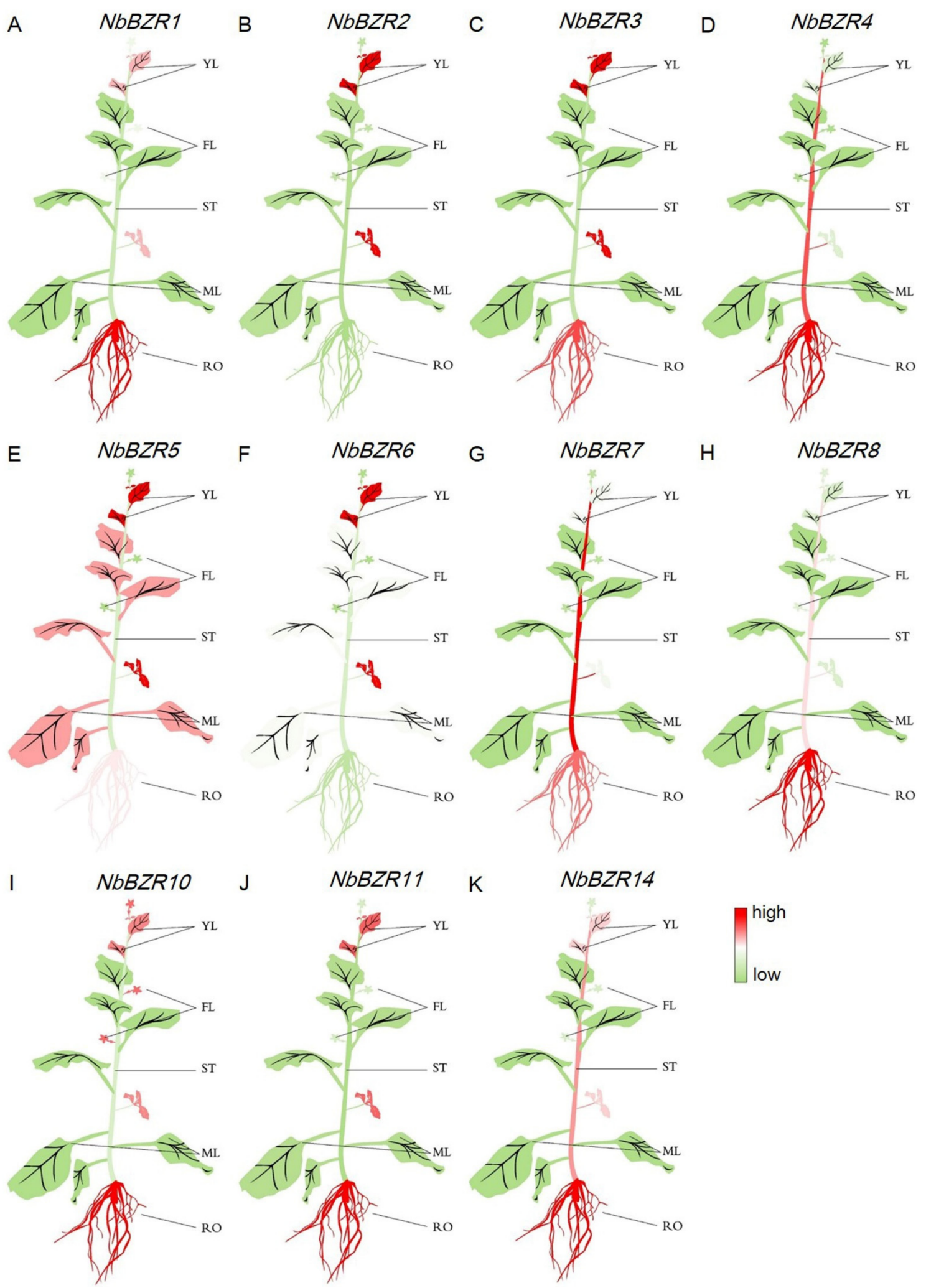

Figure 4. Expression levels of $\mathrm{NbBZRs}$ in different tissues (A-K). The mean expression values were calculated from three independent biological replicates relative to that in young leaves. YL: young leaf; MF: mature leaf; ST: stem; RO: root; FL: flower. Red represents a high expression level and green represents a low expression level. The raw data of relative expression values and standard errors are provided in Supplementary Figure S1. 


\subsection{Expression Profiles of NbBZR Genes in Response to Abiotic Stress Treatments}

We finally examined the expression patterns of the NbBZR gene family members to various types of abiotic stress: drought, cold, high temperature, and salt stress. For drought stress, samples were collected on days $0,2,4,7,10$ and 14. All 11 tested genes were significantly down-regulated after 7 days of stress treatment (Figure 5) and levels generally remained low. There was some evidence that the expression of NbBZR6 was slightly up-regulated in the early stages (2-4 days) of drought treatment (Figure 5).

NbBZR1

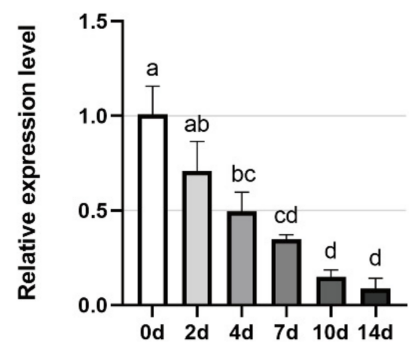

NbBZR4

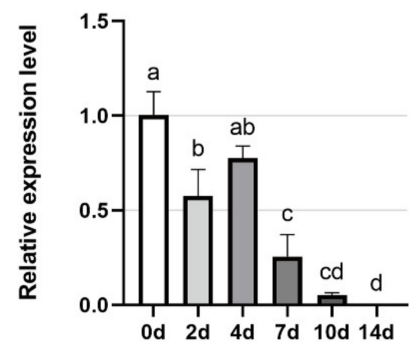

NbBZR7

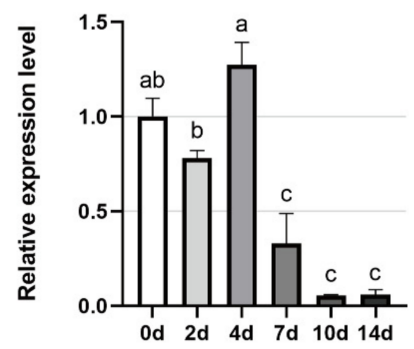

NbBZR11

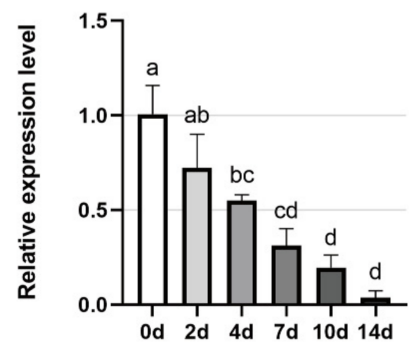

NbBZR2

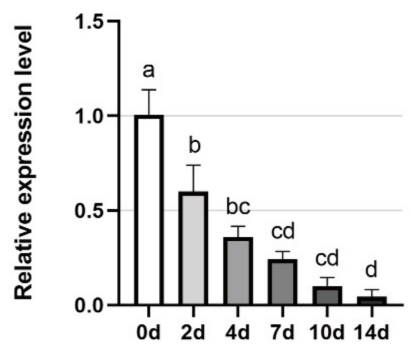

NbBZR5

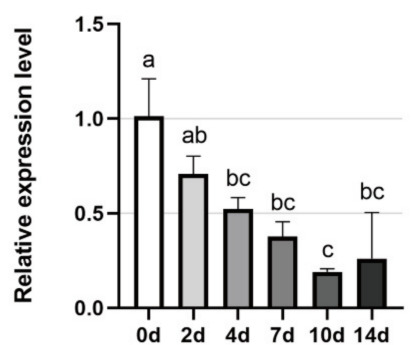

NbBZR8

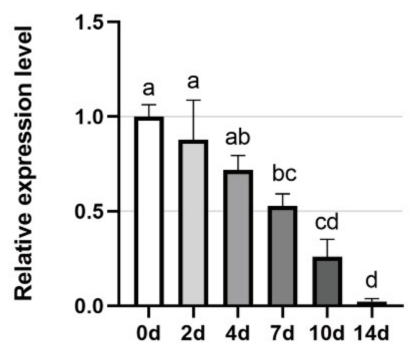

NbBZR14

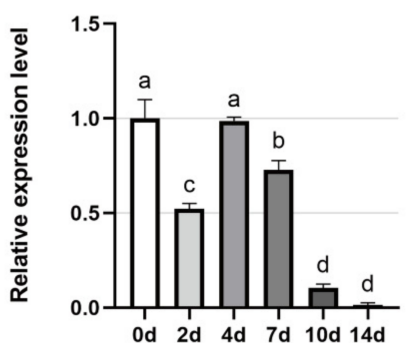

NbBZR3

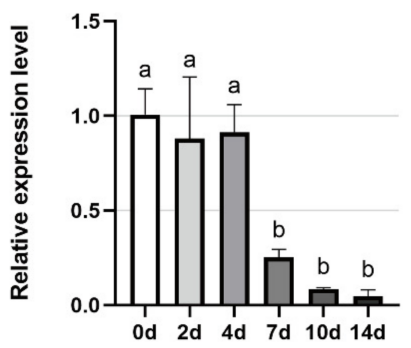

NbBZR6

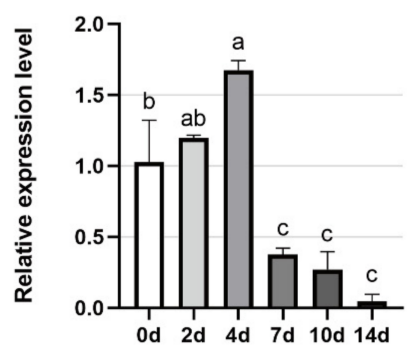

NbBZR10

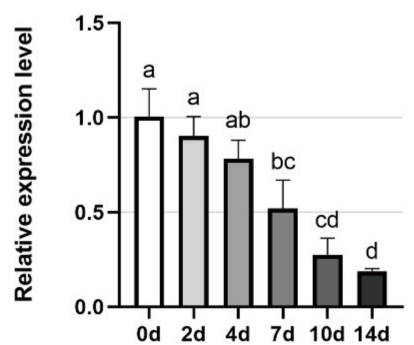

Figure 5. Expression analysis of $N b B Z R s$ after drought (0-14 days) treatment. Gene expression was normalized to control unstressed expression level, which was assigned a value of 1 . Data represent the averages of three independent experiments \pm SD. Standard errors are shown as bars above columns. Different letters indicate significant differences according to Tukey's multiple comparisons test. Columns with different marked letters are significantly different $(p<0.05)$, and those with the same marked letter or share a letter are not significantly different.

There was a complex of NbBZRs that were subject to cold treatment. NbBZRs 2, 3 and 5 were significantly up-regulated, most dramatically in NbBZR5, which showed 
a progressive up-regulation with time and was up-regulated 16-fold in $48 \mathrm{~h}$ relative to $0 \mathrm{~h}$ (Figure 6). NbBZR4/-7/-14 showed a gradual downward trend as the stress time was prolonged. NbBZR1/-8/-10/-11 were first up-regulated and then down-regulated, while NbBZR6 was first down-regulated and then up-regulated (Figure 6).

NbBZR1

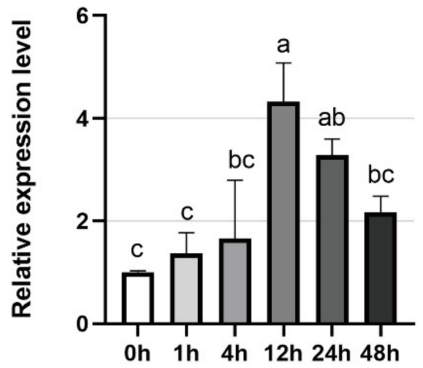

NbBZR4

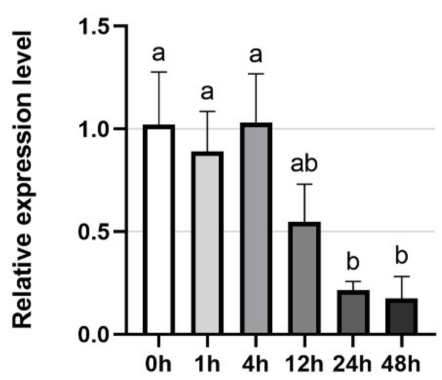

NbBZR7

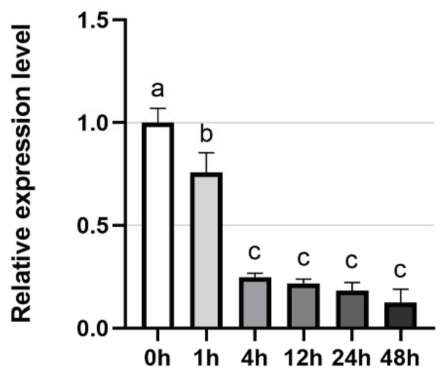

NbBZR11

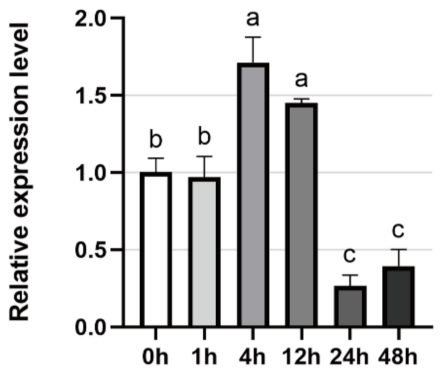

NbBZR2

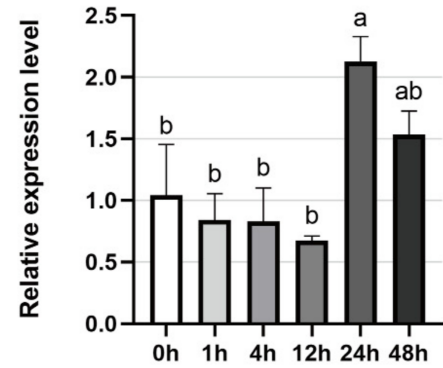

NbBZR5

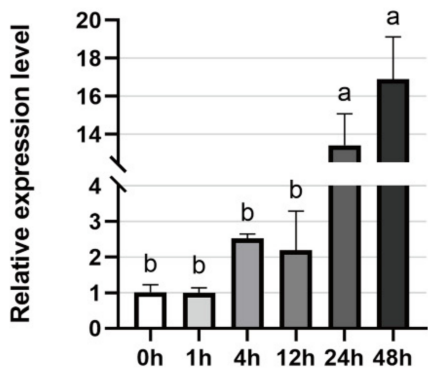

NbBZR8

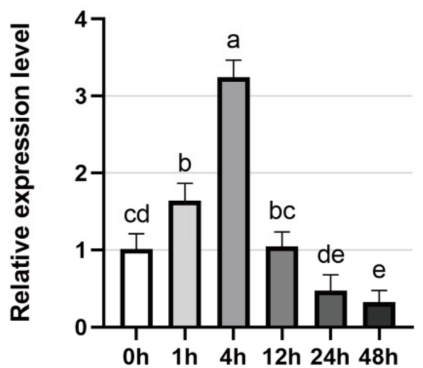

NbBZR14

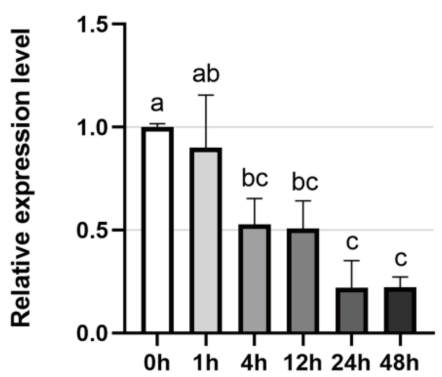

NbBZR3

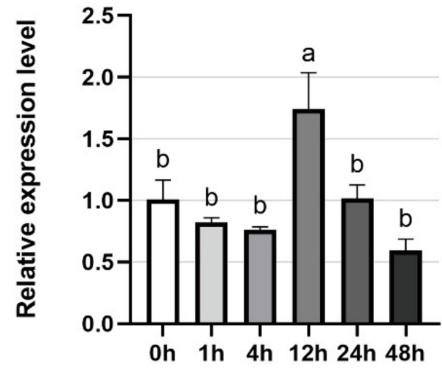

NbBZR6

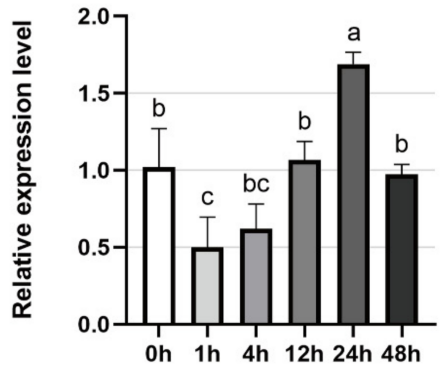

NbBZR10

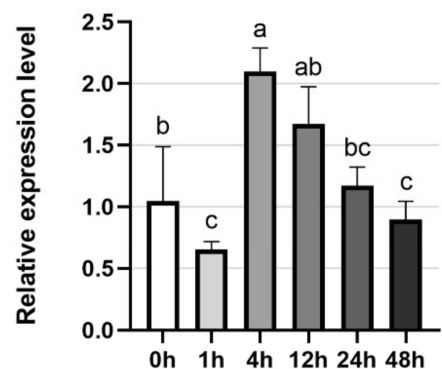

Figure 6. Expression analysis of $\mathrm{NbBZRs}$ after cold treatment (0-48 h). Gene expression was normalized to control unstressed expression level, which was assigned a value of 1 . Data represent averages of three independent experiments $\pm S D$. Standard errors are shown as bars above columns. Different letters indicate significant differences according to Tukey's multiple comparisons test. Columns with different marked letters are significantly different $(p<0.05)$, and those with the same marked letter or share a letter are not significantly different. 
During heat treatment, NbBZR1/-8/-10 were strongly and rapidly inhibited (Figure 7) while NbBZR2/-4/-6/-7 were induced to varying degrees, of which NbBZR6 was the most induced, and from an early stage (Figure 7). The levels of NbBZR3/-11 decreased on the first, second, and third days of heat treatment, and then began to increase (Figure 7).

NbBZR1

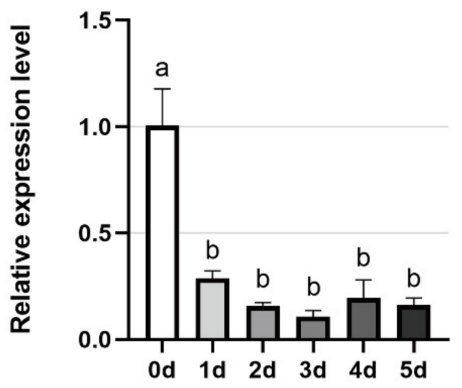

NbBZR4

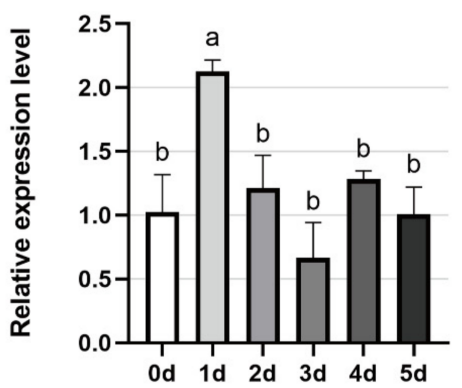

NbBZR7

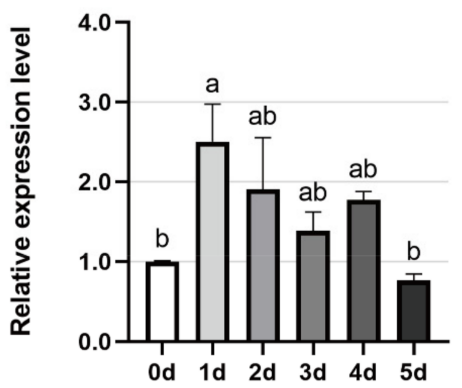

NbBZR11

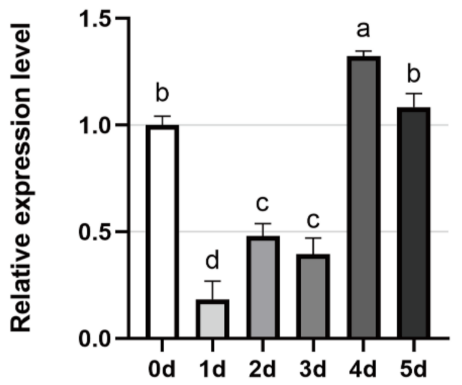

NbBZR2

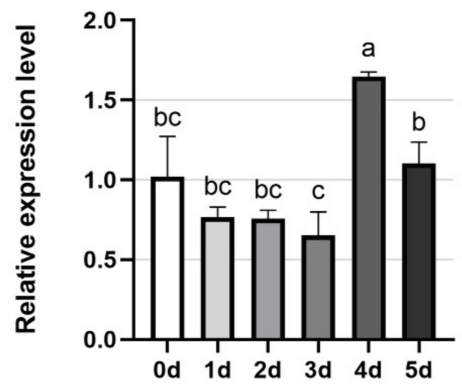

NbBZR5

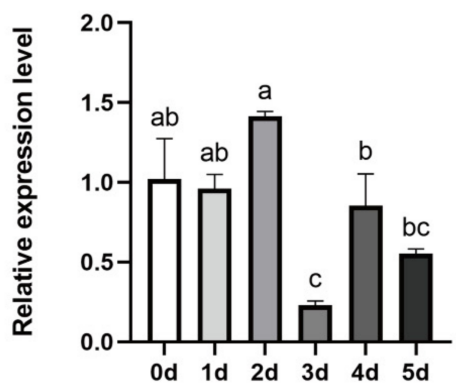

NbBZR8

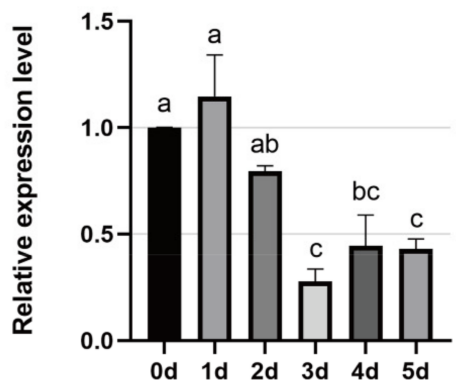

NbBZR14

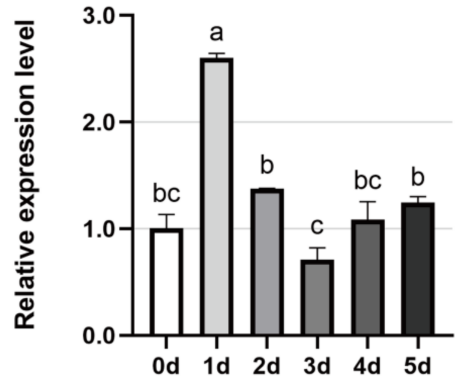

NbBZR3

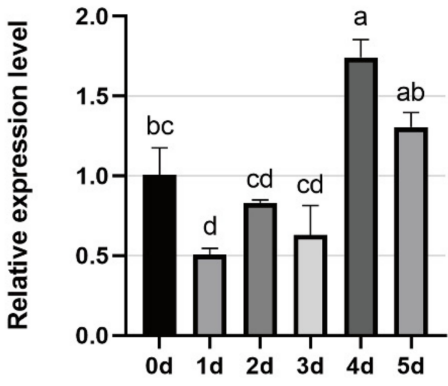

NbBZR6

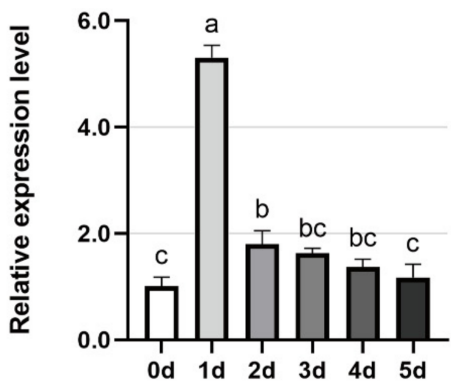

NbBZR10

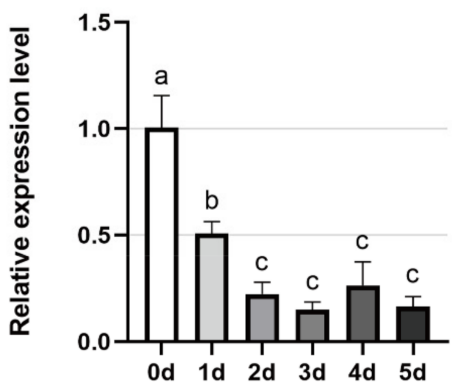

Figure 7. Expression analysis of $N b B Z R s$ after heat ( $0-5$ days) stress. Gene expression was normalized to control unstressed expression level, which was assigned a value of 1 . Data represent averages of three independent experiments \pm SD. Standard errors are shown as bars above columns. Different letters indicate significant differences according to Tukey's multiple comparisons test. Columns with different marked letters are significantly different $(p<0.05)$, and those with the same marked letter or share a letter are not significantly different. 
Under salt stress, $N b B Z R-4 /-5 /-6 /-8 /-11$ were significantly induced, especially NbBZR6 and -8 , the expression of which increased about 18-fold after $12 \mathrm{~h}$ (Figure 8). NbBZR1 was significantly down-regulated, decreasing to $20 \%$ of the control at $48 \mathrm{~h}$ (Figure 8 ). The expression levels of NbBZR14 were slightly induced after the stress treatment, while NbBZR2/-3 and -7 were slightly inhibited (Figure 8).

NbBZR1

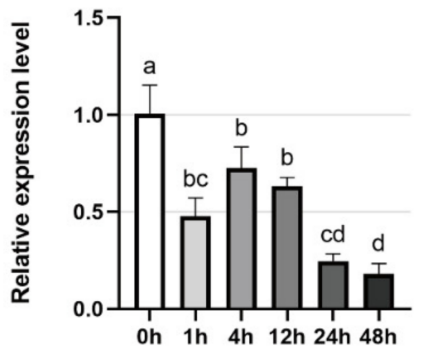

NbBZR4

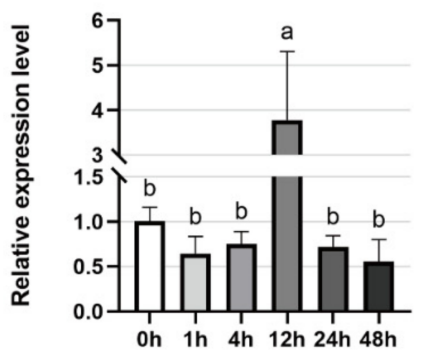

NbBZR7

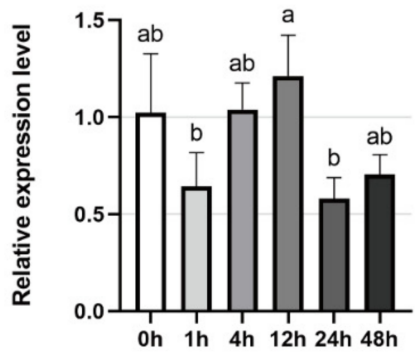

NbBZR11

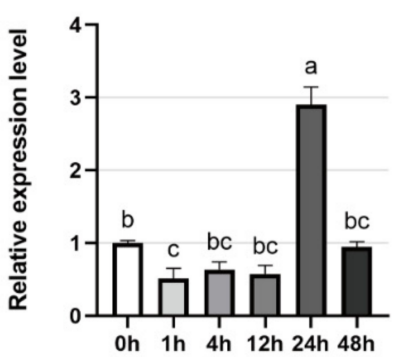

NbBZR2

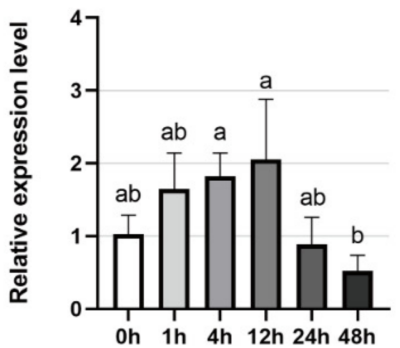

NbBZR5

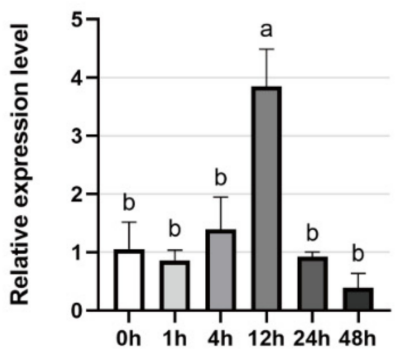

NbBZR8

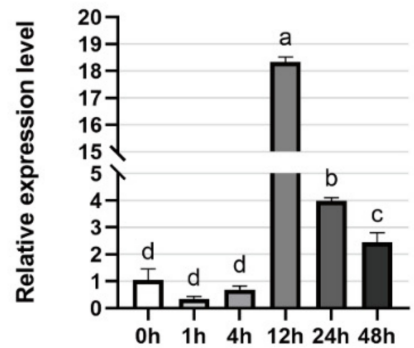

NbBZR14

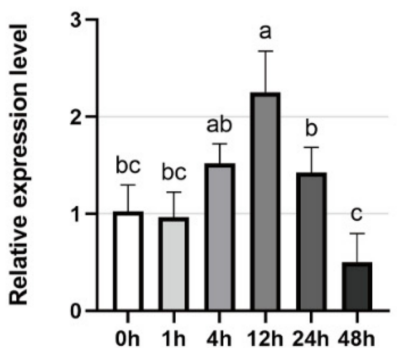

NbBZR3

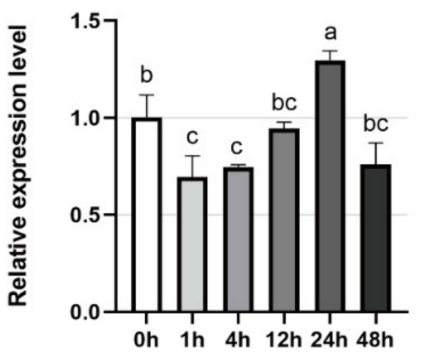

NbBZR6

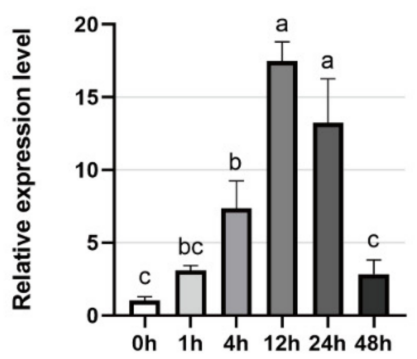

NbBZR10

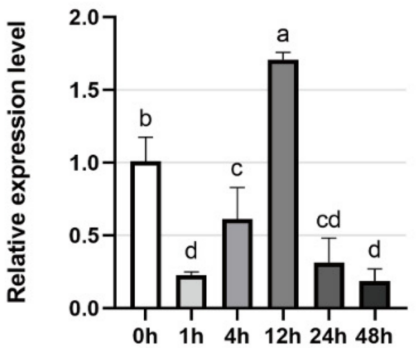

Figure 8. Expression analysis of $N b B Z R s$ after salt $(0-48 \mathrm{~h})$ treatment. Gene expression was normalized to control the unstressed expression level, which was assigned a value of 1 . Data represent averages of three independent experiments \pm SD. Standard errors are shown as bars above columns. Different letters indicate significant differences according to Tukey's multiple comparisons test. Columns with different marked letters are significantly different $(p<0.05)$, and those with the same marked letter or share a letter are not significantly different. 


\section{Discussion}

The BZR family is a small family of plant-specific transcription factors with important functions in plant growth and development and in BZR-mediated abiotic stress [30]. The BZR gene family members were systematically identified in Arabidopsis [13,14], Brassica rapa [31], sugar beet (Beta vulgaris L.) [33], maize (Zea mays L.) [32], apple (Malus domestica) [34], wheat (Triticum aestivum L.) [35] and seven legumes [30] but, prior to our study, there was no in-depth research report on the BZR TF family in N. benthamiana. In this work, a total of 14 BZR genes in the $N$. benthamiana genome were identified (Table 1). The 11 genes studied in detail were widely expressed at different levels in different tissues (Figure 4), suggesting that they may act as growth regulators. An analysis of the promoter region of the NbBZR gene revealed the existence of a variety of cis-acting elements to regulate the temporal and spatial expression levels of the genes (Figure 3). In addition to hormonal response elements, some elements related to stress and development were also identified (Figure 3). Following drought, cold, heat and salt stress, there were differential expression patterns among the NbBZR transcription factors (Figures 5-8). Thus, the NbBZR gene may be a complex participant in the regulation of plant development and abiotic stress resistance pathways.

The transcript accumulation of all 11 tested genes was significantly down-regulated after 7 days of drought stress (Figure 5), so NbBZR may respond to drought stress in a negative manner. Previous studies showed that drought stress induces the production of ABA, which promotes stress tolerance [36], and that the BR signal and ABA pathways antagonize each other to coordinate plant growth and drought stress [37,38]. Therefore, the drought-induced, down-regulation of NbBZR expression may be the result of the activation of the ABA pathway. In addition, a previous study confirmed that the BR signaling pathway inhibits the drought response by regulating the mutual inhibition mechanism between BES1 and RD26 [21]. RD26 is a negative regulator of the BR pathway, and RD26 can be induced by drought to promote the expression of drought-regulating genes, thereby enhancing the drought resistance of plants. BES1 can inhibit the expression of drought-related genes such as RD26, leading to the suppression of drought response [21]. In our results, we observed that drought stress which inhibited NbBZR gene expression may also be caused by the induction of $R D 26$. Therefore, we speculate that the knockdown of the BZR gene family of $N$. benthamiana may improve the drought stress resistance of plants. However, not all $B Z R$ genes in all species can be down-regulated under drought stress. For example, only one of the three BZR members tested after drought stress is down-regulated, while the other two are up-regulated in soybean [30]. In Medicago, among the six genes tested after drought stress, only two genes were down-regulated (MtBZR2/5), and the other genes had no effect [30]. In Brassica rapa, the expression of $10 \mathrm{BrBZR}$ genes was up-regulated after drought stress, while only two genes continued to be down-regulated during the stress treatment [31]. It shows that the BZR genes of different species respond differently to the same kind of stress.

The response of $N b B Z R$ to other stresses (cold stress, heat stress, salt stress) is irregular, some are induced, and some are inhibited, which is consistent with the phenomenon observed in other species [30-32,34,35]. In our results, members with specific responses were observed in each stress. For example, NbBZR5 increased 16-fold in $48 \mathrm{~h}$ under cold stress (Figure 6), and NbBZR1/-10 was strongly inhibited under heat stress (Figure 7). During salt stress, NbBZR6/-8 increased 18-fold in $12 \mathrm{~h}$ (Figure 8). These gene members may have important functions in the corresponding stress process and should be given priority in further functional identification.

The analysis of the expression results suggests that some $N b B Z R$ members participate in the response to multiple stresses. For example, the expression of NbBZR1 was significantly inhibited under both heat and salt stress, whereas NbBZR4/-6 were significantly induced under these treatments (Figures 7 and 8). The expression of NbBZR5 was upregulated under both cold stress and salt stress (Figures 6 and 8). It also appears that some members may respond in opposite ways to different abiotic stresses. For example, heat 
stress can inhibit the expression of NbBZR8 (Figure 7), while salt stress can increase its expression (Figure 8). In a similar manner, cold stress can down-regulate the expression levels of NbBZR4/-7/-14, while heat stress can induce them (Figures 6 and 7).

In this study, ten conservative motifs of NbBZRs are shown, and the motif composition of members in the same group are more similar (Figure 2). Motif-1 is an atypical bHLH DNA binding domain, which is the most conserved region in the BZR proteins, and the common motif in all BZR proteins [12]. Motif-4 has a serine-rich sequence (SxxxSxxxSxxxSxxxS), which is considered to be a putative phosphorylation site for members of the GSK3 kinase family [39] and is also found in the BZR genes of Legume [30], Brassica rapa [31], and wheat [35]. Motif- 8 is a PEST domain involved in the control of protein stability [12] and was also identified in the BZR genes of Legume [30] and Brassica rapa [31]. Motif-2 was identified in the BZR genes of apple [34] and Zea mays L. [32], and Motif-3/-5/-6/-10 were also found in the BZR genes of Brassica rapa [31].

In summary, these data suggest that the BZR transcription factor family in N. benthamiana plays a role in regulating plant physiology and morphology, and also has stress signal activity. The genome-wide identification and characterization of the members of the BZR TF family in N. benthamiana is an important starting point for the further in-depth study of the function of this gene family and can lay a foundation for the breeding and genetic improvement of solanaceous crops.

\section{Materials and Methods}

\subsection{NbBZR Gene Identification}

The protein sequences of the six Arabidopsis BZR genes were downloaded from The Arabidopsis Information Resource (https: / / www.arabidopsis.org/ accessed on 22 September 2021), and the genome of N. benthamiana was downloaded from the Sol Genomics Network (https://solgenomics.net/ accessed on 22 September 2021) [40]. The method used to identify all the putative NbBZR referred to Wu et al. [41]. First, all Arabidopsis BZR sequences were used to search for possible NbBZR sequences using TBtools [42]. Then, NCBI's Batch CD-Search function was used to confirm whether the candidate NbBZR had a characteristic BES1_N superfamily domain (c105316). Those candidates that did not meet the above conditions were eliminated. The predicted CDS length, isoelectric point, and molecular weight of the NbBZR genes were determined by ExPASy [43].

\subsection{Phylogenetic Analysis}

Phylogenetic analysis was performed based on the full-length protein sequences of AtBZR and NbBZR using the MEGA X program by the neighbor-joining (NJ) method, and a bootstrap test was carried out with 1000 iterations [44].

\subsection{Analysis of Conserved Motifs, Gene Structure and Functional Domains}

The conserved motifs of the genes were analyzed by the MEME program with a maximum number of motifs set to 10 [45]. Gene structures were analyzed and visualized by TBtools using $N$. benthamiana genome annotation file. Functional domains were analyzed and visualized using NCBI Batch CD-Search (https:/ / www.ncbi.nlm.nih.gov/Structure/ bwrpsb/bwrpsb.cgi accessed on 22 September 2021) and TBtools (V1.089).

\subsection{Prediction of Cis-Elements in the Putative Promoter Regions}

To investigate cis-elements in the promoter regions of all the obtained genes, we downloaded $2 \mathrm{~kb}$ of the genomic DNA sequences, upstream of the initiation codon (ATG) of each gene, from the Solanaceae genome database. The putative cis-regulatory elements in the promoter sequences were analyzed via PlantCARE (http:/ /bioinformatics.psb.ugent. be/webtools/plantcare/html/ accessed on 22 September 2021) [46] and visualized by TBtools (V1.089). 


\subsection{Plant Materials and Abiotic Stress Treatments}

In order to perform quantitative PCR expression analysis, $N$. benthamiana seeds were planted in greenhouse soil under the following controlled conditions: temperature $23 \pm 1{ }^{\circ} \mathrm{C}, 14 \mathrm{~h}$ light $/ 10 \mathrm{~h}$ night, and relative humidity $60 \%$. N. benthamiana was transferred to stress treatment 21 days after planting. Drought stress consisted of stopping the normal watering regime for $14 \mathrm{~d}$ and leaves were sampled $0,2,4,7,10$ and 14 days after the treatment started [30]. For salt stress, the plants were treated with $200 \mathrm{mM} \mathrm{NaCl}$ solution and the leaves were collected at $0,1,4,12,24$ and $48 \mathrm{~h}$ after salt treatment [30]. For cold or heat treatment, the plants were subjected to $4 \pm 1^{\circ} \mathrm{C}$ [31] or $30 \pm 1{ }^{\circ} \mathrm{C}$ [47] conditions, respectively. The leaves were collected at $0,1,4,12,24$ and $48 \mathrm{~h}$ after cold treatment [31] and at $0,1,2,3,4$ and $5 \mathrm{~d}$ after heat treatment $[47,48]$. All collected samples from three biological replicates were quickly frozen in liquid nitrogen and stored at $-80^{\circ} \mathrm{C}$. RNA was extracted to analyze the expression patterns of the different BZR transcription factors.

\subsection{RNA Isolation and Expression Analysis of NbBZR Genes}

Total RNA was extracted by the TRIZOL method, and the first cDNA strand was generated using Toyobo cDNA First Strand Synthesis Kit (Toyobo, Shanghai, China). Then, the RT-qPCR was performed on the Roche LightCycler ${ }^{\circledR} 480$ Real-Time PCR instrument (Rotkreuz, Switzerland) with Toyobo Premix Kit (Toyobo, Shanghai, China). Three independent biological replicates and three technical replicates were adopted. The N. benthamiana ubiquitin-conjugating enzyme E2 (UBC) gene was used as the internal reference gene to calculate relative gene expression levels using the $2^{-\Delta \Delta C(t)}$ method [49]. All primers are listed in the Supplementary Table S4.

\section{Conclusions}

This study systematically identified the BZR TF members in N. benthamiana, and analyzed their tissue-specific expression and expression profiles in response to four abiotic stresses. The results indicated that $N b B Z R$ may have had a variety of resistance-related functions. Therefore, our findings in this study will help to select suitable candidate genes for further functional identification.

Supplementary Materials: The following are available online at https:/ /www.mdpi.com/article/10 $.3390 /$ ijms221910379/s1.

Author Contributions: Conceptualization, S.R. and F.Y.; methodology, H.Z., Y.L., J.P. and G.W.; investigation, X.C., X.W. and S.Q.; writing-original draft preparation, S.R.; writing-review and editing, F.Y.; supervision, F.Y.; funding acquisition, F.Y. and J.C. All authors have read and agreed to the published version of the manuscript.

Funding: This work was financially supported by the Chinese Agriculture Research System (CARS24-C-04, from January 2019) and K. C. Wong Magna Fund in Ningbo University (from January 2018). The funders did not participate in the design of this study, data collection, analysis, and manuscript writing.

Institutional Review Board Statement: Not applicable.

Informed Consent Statement: Not applicable.

Acknowledgments: We thank M. J. Adams, Minehead, UK, for critical reading of the manuscript.

Conflicts of Interest: The authors declare no conflict of interest. The funders had no role in the design of the study; in the collection, analyses, or interpretation of data; in the writing of the manuscript, or in the decision to publish the results. 


\section{References}

1. Nolan, T.M.; Vukašinović, N.; Liu, D.; Russinova, E.; Yin, Y. Brassinosteroids: Multidimensional regulators of plant growth, development, and stress responses. Plant Cell 2020, 32, 295-318. [CrossRef]

2. Kim, T.W.; Guan, S.; Sun, Y.; Deng, Z.; Tang, W.; Shang, J.X.; Sun, Y.; Burlingame, A.L.; Wang, Z.Y. Brassinosteroid signal transduction from cell-surface receptor kinases to nuclear transcription factors. Nat. Cell Biol. 2009, 11, 1254-1260. [CrossRef] [PubMed]

3. Clouse, S.D. Brassinosteroid signal transduction: From receptor kinase activation to transcriptional networks regulating plant development. Plant Cell 2011, 23, 1219-1230. [CrossRef] [PubMed]

4. Guo, H.; Li, L.; Aluru, M.; Aluru, S.; Yin, Y. Mechanisms and networks for Brassinosteroid regulated gene expression. Curr. Opin. Plant Biol. 2013, 16, 545-553. [CrossRef] [PubMed]

5. Nolan, T.; Chen, J.; Yin, Y. Cross-talk of Brassinosteroid signaling in controlling growth and stress responses. Biochem. J. 2017, 474, 2641-2661. [CrossRef]

6. Kim, T.W.; Wang, Z.Y. Brassinosteroid signal transduction from receptor kinases to transcription factors. Annu. Rev. Plant Biol. 2010, 61, 681-704. [CrossRef]

7. Li, J.; Nam, K.H.; Vafeados, D.; Chory, J. BIN2, a new Brassinosteroid-insensitive locus in Arabidopsis. Plant Physiol. 2001, 127, 14-22. [CrossRef]

8. Sun, Y.; Fan, X.-Y.; Cao, D.-M.; Tang, W.; He, K.; Zhu, J.-Y.; He, J.-X.; Bai, M.-Y.; Zhu, S.; Oh, E.; et al. Integration of Brassinosteroid signal transduction with the transcription network for plant growth regulation in Arabidopsis. Dev. Cell 2010, $19,765-777$. [CrossRef]

9. Tang, W.; Yuan, M.; Wang, R.; Yang, Y.; Wang, C.; Oses-Prieto, J.A.; Kim, T.-W.; Zhou, H.-W.; Deng, Z.; Gampala, S.S.; et al. PP2A activates Brassinosteroid-responsive gene expression and plant growth by dephosphorylating BZR1. Nat. Cell Biol. 2011, 13, 124-131. [CrossRef]

10. Yu, X.; Li, L.; Zola, J.; Aluru, M.; Ye, H.; Foudree, A.; Guo, H.; Anderson, S.; Aluru, S.; Liu, P.; et al. A Brassinosteroid transcriptional network revealed by genome-wide identification of BESI target genes in Arabidopsis thaliana. Plant J. 2011, 65, 634-646. [CrossRef]

11. Li, L.; Deng, X.W. It runs in the family: Regulation of Brassinosteroid signaling by the BZR1-BES1 class of transcription factors. Trends Plant Sci. 2005, 10, 266-268. [CrossRef]

12. Yin, Y.; Vafeados, D.; Tao, Y.; Yoshida, S.; Asami, T.; Chory, J. A new class of transcription factors mediates Brassinosteroidregulated gene expression in Arabidopsis. Cell 2005, 120, 249-259. [CrossRef]

13. Wang, Z.Y.; Nakano, T.; Gendron, J.; He, J.; Chen, M.; Vafeados, D.; Yang, Y.; Fujioka, S.; Yoshida, S.; Asami, T.; et al. Nuclearlocalized BZR1 mediates Brassinosteroid-induced growth and feedback suppression of Brassinosteroid biosynthesis. Dev. Cell 2002, 2, 505-513. [CrossRef]

14. Yin, Y.; Wang, Z.-Y.; Mora-Garcia, S.; Li, J.; Yoshida, S.; Asami, T.; Chory, J. BES1 accumulates in the nucleus in response to Brassinosteroids to regulate gene expression and promote stem elongation. Cell 2002, 109, 181-191. [CrossRef]

15. Bechtold, U.; Field, B. Molecular mechanisms controlling plant growth during abiotic stress. J. Exp. Bot. 2018, 69, 2753-2758. [CrossRef] [PubMed]

16. Feng, W.; Lindner, H.; Robbins, N.N.; Dinneny, J.R. Growing Out of Stress: The role of cell- and organ-scale growth control in plant water-stress responses. Plant Cell 2016, 28, 1769-1782. [CrossRef]

17. Yoshida, T.; Mogami, J.; Yamaguchi-Shinozaki, K. ABA-dependent and ABA-independent signaling in response to osmotic stress in plants. Curr. Opin. Plant Biol. 2014, 21, 133-139. [CrossRef] [PubMed]

18. Zhu, Y.; Wang, B.; Tang, K.; Hsu, C.C.; Xie, S.; Du, H.; Yang, Y.; Tao, W.A.; Zhu, J.-K. An Arabidopsis Nucleoporin NUP85 modulates plant responses to ABA and salt stress. PLoS Genet. 2017, 13, e1007124. [CrossRef]

19. Planas-Riverola, A.; Gupta, A.; Betegón-Putze, I.; Bosch, N.; Ibañes, M.; Caño-Delgado, A.I. Brassinosteroid signaling in plant development and adaptation to stress. Development 2019, 146, dev151894. [CrossRef] [PubMed]

20. Nolan, T.M.; Brennan, B.; Yang, M.; Chen, J.; Zhang, M.; Li, Z.; Wang, X.; Bassham, D.C.; Walley, J.; Yin, Y. Selective autophagy of BES1 mediated by DSK2 balances plant growth and survival. Dev. Cell 2017, 41, 33-46.e7. [CrossRef] [PubMed]

21. Ye, H.; Liu, S.; Tang, B.; Chen, J.; Xie, Z.; Nolan, T.M.; Jiang, H.; Guo, H.; Lin, H.-Y.; Li, L.; et al. RD26 mediates crosstalk between drought and Brassinosteroid signaling pathways. Nat. Commun. 2017, 8, 14573. [CrossRef]

22. Jiang, H.; Tang, B.; Xie, Z.; Nolan, T.; Ye, H.; Song, G.; Walley, J.; Yin, Y. GSK3-like kinase BIN2 phosphorylates RD26 to potentiate drought signaling in Arabidopsis. Plant J. 2019, 100, 923-937. [CrossRef]

23. Xie, Z.; Nolan, T.; Jiang, H.; Tang, B.; Zhang, M.; Li, Z.; Yin, Y. The AP2/ERF transcription factor TINY modulates Brassinosteroidregulated plant growth and drought responses in Arabidopsis. Plant Cell 2019, 31, 1788-1806. [CrossRef]

24. Chen, J.; Nolan, T.; Ye, H.; Zhang, M.; Tong, H.; Xin, P.; Chu, J.; Chu, C.; Li, Z.; Yin, Y. Arabidopsis WRKY46, WRKY54, and WRKY70 transcription factors are involved in Brassinosteroid-regulated plant growth and drought responses. Plant Cell 2017, 29, 1425-1439. [CrossRef]

25. Li, H.; Ye, K.; Shi, Y.; Cheng, J.; Zhang, X.; Yang, S. BZR1 positively regulates freezing tolerance via CBF-dependent and CBF-independent pathways in Arabidopsis. Mol. Plant 2017, 10, 545-559. [CrossRef] [PubMed] 
26. Ibañez, C.; Delker, C.; Martinez, C.; Bürstenbinder, K.; Janitza, P.; Lippmann, R.; Ludwig, W.; Sun, H.; James, G.V.; Klecker, M.; et al. Brassinosteroids dominate hormonal regulation of plant thermomorphogenesis via BZR1. Curr. Biol. 2018, 28, 303-310.e3. [CrossRef] [PubMed]

27. Oh, E.; Zhu, J.Y.; Wang, Z.Y. Interaction between BZR1 and PIF4 integrates Brassinosteroid and environmental responses. Nat. Cell Biol. 2012, 14, 802-809. [CrossRef] [PubMed]

28. Geng, Y.; Wu, R.; Wee, C.W.; Xie, F.; Wei, X.; Chan, P.M.; Tham, C.; Duan, L.; Dinneny, J.R. A spatio-temporal understanding of growth regulation during the salt stress response in Arabidopsis. Plant Cell 2013, 25, 2132-2154. [CrossRef]

29. Cui, X.-Y.; Gao, Y.; Guo, J.; Yu, T.-F.; Zheng, W.-J.; Liu, Y.-W.; Chen, J.; Xu, Z.-S.; Ma, Y.-Z. BES/BZR transcription factor TaBZR2 positively regulates drought responses by activation of TaGST1. Plant Physiol. 2019, 180, 605-620. [CrossRef] [PubMed]

30. Li, Y.; He, L.; Li, J.; Chen, J.; Liu, C. Genome-wide identification, characterization, and expression profiling of the legume BZR transcription factor gene family. Front. Plant Sci. 2018, 9, 1332.

31. Saha, G.; Park, J.-I.; Jung, H.-J.; Ahmed, N.U.; Kayum, A.; Kang, J.-G.; Nou, I.-S. Molecular characterization of BZR transcription factor family and abiotic stress induced expression profiling in Brassica rapa. Plant Physiol. Biochem. 2015, 92, 92-104. [CrossRef] [PubMed]

32. Manoli, A.; Trevisan, S.; Quaggiotti, S.; Varotto, S. Identification and characterization of the BZR transcription factor family and its expression in response to abiotic stresses in Zea mays L. Plant Growth Regul. 2018, 84, 423-436. [CrossRef]

33. Wang, W.; Sun, Y.Q.; Li, G.L.; Zhang, S.Y. Genome-wide identification, characterization, and expression patterns of the BZR transcription factor family in sugar beet (Beta vulgaris L.). BMC Plant Biol. 2019, 19, 191. [CrossRef]

34. Cao, X.; Khaliq, A.; Lu, S.; Xie, M.; Ma, Z.; Mao, J.; Chen, B. Genome-wide identification and characterization of the BES1 gene family in apple (Malus domestica). Plant Biol. 2020, 22, 723-733. [CrossRef]

35. Kesawat, M.S.; Kherawat, B.S.; Singh, A.; Dey, P.; Kabi, M.; Debnath, D.; Khandual, A.; Rout, S.; Ali, A.; Palem, R.R. Genome-wide identification and characterization of the Brassinazole-resistant (BZR) gene family and its expression in the various developmental stage and stress conditions in Wheat (Triticum aestivum L.). Int. J. Mol. Sci. 2021, 22, 8743. [CrossRef]

36. Cutler, S.R.; Rodriguez, P.L.; Finkelstein, R.R.; Abrams, S.R. Abscisic acid: Emergence of a core signaling network. Annu. Rev. Plant Biol. 2010, 61, 651-679. [CrossRef]

37. Ryu, H.; Cho, H.; Bae, W.; Hwang, I. Control of early seedling development by BES1/TPL/HDA19-mediated epigenetic regulation of ABI3. Nat. Commun. 2014, 5, 4138. [CrossRef] [PubMed]

38. Yang, X.; Bai, Y.; Shang, J.; Xin, R.; Tang, W. The antagonistic regulation of abscisic acid-inhibited root growth by Brassinosteroids is partially mediated via direct suppression of ABSCISIC ACID INSENSITIVE 5 expression by BRASSINAZOLE RESISTANT 1. Plant Cell Environ. 2016, 39, 1994-2003. [CrossRef]

39. Li, J.; Nam, K.H. Regulation of brassinosteroid signaling by a GSK3/SHAGGY-like kinase. Science 2002, 295, 1299-1301. [PubMed]

40. Bombarely, A.; Rosli, H.G.; Vrebalov, J.; Moffett, P.; Mueller, L.A.; Martin, G.B. A draft genome sequence of Nicotiana benthamiana to enhance molecular plant-microbe biology research. Mol. Plant Microbe Interact. 2012, 25, 1523-1530. [CrossRef]

41. Wu, X.; Lai, Y.; Lv, L.; Ji, M.; Han, K.; Yan, D.; Lu, Y.; Peng, J.; Rao, S.; Yan, F.; et al. Fasciclin-like arabinogalactan gene family in Nicotiana benthamiana: Genome-wide identification, classification and expression in response to pathogens. BMC Plant Biol. 2020, 20, 305. [CrossRef] [PubMed]

42. Chen, C.; Chen, H.; Zhang, Y.; Thomas, H.R.; Frank, M.H.; He, Y.; Xia, R. TBtools: An integrative toolkit developed for interactive analyses of big biological data. Mol. Plant 2020, 13, 1194-1202. [CrossRef]

43. Wilkins, M.R.; Gasteiger, E.; Bairoch, A.; Sanchez, J.-C.; Williams, K.L.; Appel, R.D.; Hochstrasser, D.F. Protein identification and analysis tools in the ExPASy server. Methods Mol. Biol. 1999, 112, 531-552. [PubMed]

44. Kumar, S.; Stecher, G.; Li, M.; Knyaz, C.; Tamura, K. MEGA X: Molecular evolutionary genetics analysis across computing platforms. Mol. Biol. Evol. 2018, 35, 1547-1549. [CrossRef]

45. Bailey, T.L.; Boden, M.; Buske, F.A.; Frith, M.; Grant, C.E.; Clementi, L.; Ren, J.; Li, W.W.; Noble, W.S. MEME SUITE: Tools for motif discovery and searching. Nucleic Acids Res. 2009, 37, W202-W208. [CrossRef]

46. Lescot, M.; Déhais, P.; Thijs, G.; Marchal, K.; Moreau, Y.; Van de Peer, Y.; Rouzé, P.; Rombauts, S. PlantCARE, a database of plant cis-acting regulatory elements and a portal to tools for in silico analysis of promoter sequences. Nucleic Acids Res. 2002, 30, 325-327. [CrossRef]

47. Zhao, P.; Wang, D.; Wang, R.; Kong, N.; Zhang, C.; Yang, C.; Wu, W.; Ma, H.; Chen, Q. Genome-wide analysis of the potato Hsp20 gene family: Identification, genomic organization and expression profiles in response to heat stress. BMC Genom. $2018,19,61$. [CrossRef]

48. Martínez, C.; Espinosa-Ruíz, A.; de Lucas, M.; Bernardo-García, S.; Franco-Zorrilla, J.M.; Prat, S. PIF4-induced BR synthesis is critical to diurnal and thermomorphogenic growth. EMBO J. 2018, 37, e99552. [CrossRef]

49. Livak, K.J.; Schmittgen, T.D. Analysis of relative gene expression data using real-time quantitative PCR and the $2^{-\Delta \Delta C(t)}$ method. Methods 2001, 25, 402-408. [CrossRef] 